\title{
Clifford Rosen
}

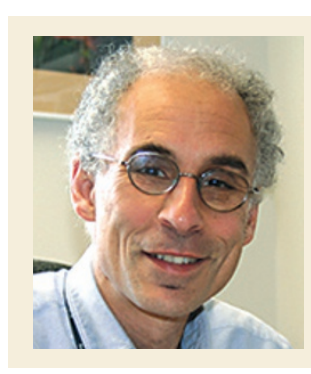

Director of Clinical and Translational Research, and a Senior Staff Scientist at Maine Medical Center, USA. In addition to his responsibilities at Maine Medical Center, Clifford Rosen is a Senior Staff Scientist at the Jackson Laboratory and Professor of Nutrition at the University of Maine, Orono. Rosen has overseen numerous Phase II and III clinical trials, funded both privately and through the National Institutes of Health. He is a member of the FDA Endocrinologic and Metabolic Drugs Advisory Committee and a former chairperson of that committee.

Recently the FDA issued draft guidance for the development of drugs and therapeutic biologics for the treatment and prevention of diabetes mellitus. What did they recommend and why?

Overall, it appears that the FDA is starting to look for more than glycaemic durability as a major outcome prior to approval. The guidance is moving towards asking sponsors for more patient-specific outcome data at the time of approval or subsequent to approval if a surrogate end point (a biomarker intended to substitute for a clinical end point) was used in the clinical-trial data submitted for approval. Examples of such patient-specific outcome data include amputation, stroke and peripheral neuropathy that are subjectively important for the patient and their quality of life but also measure significant outcomes.

I think that the FDA is asking sponsors to be more cognizant of the fact that new drugs may have side effects or profiles in clinical practice beyond what is seen in animal data, and that longer studies will be necessary for approval if there are preclinical issues. Even if there are no early warning signals there could still be long-term side effects, so the FDA would expect sponsors to provide information to support or refute the need for longer studies.

How have surrogate end points been used by the FDA to approve diabetes drugs and what is your view of their use?

For drugs indicated to treat type 2 diabetes, the FDA has used both short- and long-term changes in haemoglobin A1c (HbA1c) as their main indicator of glycaemic durability, while of course mandating safety as the key determinant for approval.

For those with type 1 diabetes we have fairly strong evidence that good glycaemic control not only reduces angiopathic disease processes such as kidney and eye disease but also reduces long-term cardiovascular outcomes. However, for type 2 diabetes, the $\mathrm{HbA1c}$ surrogate end point may not capture the entire risk profile for cardiovascular disease, as there are multiple factors such as lipid control, genetics and weight that practitioners also need to focus on.

My goal would be to set a new standard such that if glycaemic durability is used alone, then the drug should get conditional approval. It would then place the responsibility on the sponsor to do other Phase III studies of specific outcomes to get final approval for the drug. This way the drug is available and the limitations of glycaemic durability are known to the patient and the provider. Conditional approval may also help to prevent the widespread advertisement and consumer barrage that occurs when the new drugs come out before they are really well vetted in terms of side effects and other long-term risk factors.

How will the draft guidance affect companies with diabetes mellitus products currently in their clinical development programmes? Some companies have already started planning long-term Phase III trials when they come to the FDA for a new drug approval. Those that have not are going to realize that glycaemic durability alone will not be sufficient. This will have an economic impact because patient-specific outcome trials are extremely expensive, partially due to the higher patient numbers required and the extended trial length. Sponsors will also have to focus on how adverse events are identified and adjudicated - it is not going to be sufficient with a drug such as a glitazone to record chest pain as an adverse event unless there is a follow-up evaluation. These things take a lot of time and are very expensive.
Is it possible to balance the need for novel agents with the need to show that surrogate end points are clinically relevant without raising the bar too high and discouraging sponsors?

Sponsors should go to the FDA if they see a signal in their animal or Phase II studies with a plan to address any issue. In my ideal world that would mean sponsors could get conditional approval. However, it would not be wise to consider advertising the drug as if it is the 'be all and end all' based on a surrogate marker, but rather conduct the right and appropriate trials to address important patient-specific outcomes. However, it is important not to raise the bar too high because we heard too many personal testimonies at the FDA hearing on rosiglitazone saying that it was the only drug for them that controlled glucose levels, indicating that those drugs clearly work for some patients.

\section{Are there any other major challenges for the design and conduct of clinical trials for diabetes mellitus that have not been addressed and, if so, how might they be tackled?}

The biggest challenge is trying to understand the basic pathogenesis of the disease process. Why is it that within the glitazone class, for example, you have rosiglitazone that is associated with an increased cardiovascular risk and pioglitazone, which looks very similar, but has a very different cardiovascular risk profile? What is it that contributes to these really distinctive effects within the same class of drugs? Also, recently the ACCORD trial was stopped because intensive lowering of blood sugar levels was associated with increased risk of death in type 2 diabetes patients at risk for heart attack and stroke. We need to understand why tight glucose control has such a profound negative effect on overall mortality. From an industry or sponsor point of view, getting the answers to these questions is a very difficult and timeconsuming process. More money is needed to invest in these trials and for academic institutions worldwide to investigate the basic mechanisms underlying cardiovascular risk in type 2 diabetes mellitus. 